\title{
Factors Influencing the Prescribing Preferences of Physicians for Drug-Naive Patients with Type 2 Diabetes Mellitus in the Real-World Setting in Japan: Insight from a Web Survey
}

Hiroki Murayama · Kota Imai · Masato Odawara

Received: March 14, 2018 / Published online: April 25, 2018

(C) The Author(s) 2018

\section{ABSTRACT}

Introduction: The Japanese guidelines for type 2 diabetes mellitus (T2DM) emphasize individualization of treatment based on patient need and encourage physicians to select an appropriate oral antidiabetes drug (OAD). However, limited evidence is available on the factors influencing the selection by physicians (diabetes specialists and nonspecialists) of the firstline $\mathrm{OAD}$ to treat drug-naive patients with T2DM. A survey was designed to explore the treatment factors and patient characteristics that influence physicians when they choose an initial OAD to prescribe to a drug-naive patient with T2DM in a real-world setting in Japan.

Methods: The 25-min web-based online survey consisted of simple and focused multiple-choice questions, and was circulated to physicians across eight selected regions in Japan. The

Enhanced Digital Features To view enhanced digital features for this article go to https://doi.org/10.6084/ m9.figshare.6126977.

H. Murayama $(\varangle) \cdot$ K. Imai

Medical Division, Novartis Pharma K.K, Toranomon

Hills, Mori tower, 23-1, Toranomon 1-Chome,

Minato-ku, Tokyo 105-6333, Japan

e-mail: hiroki.murayama@novartis.com

M. Odawara

Department of Diabetes, Endocrinology,

Metabolism, and Rheumatology, Tokyo Medical

University, Tokyo, Japan primary endpoints were the proportions of physicians who considered particular treatment factors and patient characteristics when selecting the appropriate treatment for drug-naive T2DM patients.

Results: A total of 491 physicians participated in the survey. Dipeptidyl peptidase- 4 inhibitors (DPP-4is) were the most-preferred first-line OADs, followed by metformin, of both specialists $(69 \%$ vs. $60 \%)$ and nonspecialists (73\% vs. $47 \%)$. The most influential factors when a DPP$4 \mathrm{i}$ was selected were found to be glycated hemoglobin (HbA1c), postprandial glucose (PPG)-lowering effect, and a low risk of hypoglycemia, which were considered by $>80 \%$ of physicians, whereas the key factors when metformin was selected were improvement in insulin resistance, low cost, low risk of hypoglycemia, and PPG- and HbA1c-lowering effects, which were considered by $>85 \%$ of physicians. Regression analysis revealed that the dominant reason for choosing DPP-4is over metformin was their ease of use in patients with renal impairment, whereas the dominant reasons for choosing metformin over DPP-4is were improvement in insulin resistance and low cost. The key patient characteristics driving the choice of DPP-4is or metformin as the first-line OAD by physicians were similar to those that influenced the treatment intensification decision (DPP-4is: PPG and renal function; metformin: age, BMI, insulin resistance, and renal function). 
Conclusion: In Japan, DPP-4is are the preferred first-line OADs, followed by metformin. The key treatment factors and patient characteristics considered when selecting DPP-4is or metformin are similar for both specialists and nonspecialists. These results may prompt further discussion of the differences in T2DM treatment between Japan and other counties.

Funding: Novartis.

Keywords: Dipeptidyl peptidase- 4 inhibitor; Diabetes nonspecialist; Diabetes specialist; Drug-naive; Japan; First line; Metformin; Online survey; Oral antidiabetes drugs; Treatment choices; Type 2 diabetes mellitus

\section{INTRODUCTION}

In parallel with the steadily increasing burden imposed by diabetes in Japan [1,2], the global landscape of type 2 diabetes mellitus (T2DM) management has evolved considerably over the last decade, especially in terms of the availability of new classes of antihyperglycemic agents (AHAs). In Japan, nine different classes of AHAs are currently approved for the treatment of T2DM, including oral antidiabetes drugs (OADs) and injectables such as insulin and glucagonlike peptide-1 (GLP-1) receptor agonists [3]. Despite the increased accessibility of all types of AHAs in Japan, less than half of all patients with T2DM reach the optimal glycemic goal of HbA1c $<7 \%$ [4-6].

The T2DM guidelines in Japan [3] recommend a patient-centered approach in which the physician (a diabetes specialist or nonspecialist) chooses a medication at their discretion, based on factors such as the patient's age, T2DM duration, complications of the patient's T2DM, risk of hypoglycemia, and support systems. In such a scenario where the guidelines emphasize tailored therapy but do not guide the physician on the application of a specific regime to drugnaive patients, the optimal management of T2DM becomes increasingly challenging and complex, especially for physicians who are not familiar with diabetes treatment. Additionally, factors such as limited time, the expanding armamentarium of OADs, comorbidities, drug costs (when patients have to pay for the drug), and polypharmacy can pose a challenge to physicians treating T2DM [7]. This could lead to wide variation in the drugs chosen and in treatment patterns among the physicians in Japan [8].

Although metformin is the first-line therapeutic option for T2DM in the US and European countries [9], a considerable number of T2DM patients receive other OADs as their initial therapy $[10,11]$, which suggests that, apart from recommendations, the pathophysiology of the disease and the patient's condition can also influence pharmacotherapy practice [12].

It is therefore vital to understand the factors that influence the selection of treatment for drug-naive T2DM patients by physicians. Although there is substantial evidence of the prescription patterns that occur in many countries, including Japan [8, 13-15], little or no data are available on the process by which specialists and nonspecialists choose the appropriate treatment for newly diagnosed T2DM patients based on therapeutic regimen and patient characteristics.

Thus, the aim of the study reported in the present paper was to understand physicians' preferences and to explore the factors influencing their choice of treatment of T2DM in the real-world setting in Japan.

\section{METHODS}

\section{Study Design}

This was a noninterventional 25-min web-based online survey for physicians, which comprised focused multiple-choice questions. The posting of free text comments was restricted in order to make the survey simple and lucid. This study did not include data collected from patients and hence did not follow a therapy protocol, a diagnostic/therapy procedure, or a visit schedule. The online questionnaire included questions on the physician's profile, the number of drug-naive patients, and the number of patients treated with each OAD. It also probed the reasons for selecting OADs for use as a first-line therapy and for treatment intensification, 
taking both treatment factors and patient characteristics into account. Participating physicians were requested to score each OAD used as first-line therapy in order of priority (maximum score: 7). The recruitment criteria for the physicians who participated in the survey included the following:

- The majority (50\% or more) of their professional time was spent in direct patient care, excluding nonclinical activities such as research or teaching

- They had personally managed/treated at least 150 patients with T2DM in the last 6 months

Diabetes specialists were defined as being board certified by the Japan Diabetes Society (JDS), whereas nonspecialists were defined as physicians who had not been board certified by the JDS, even if they had treated many patients with T2DM.

\section{Data Sources}

The survey data were collected by M3 Global Research in Japan (Tokyo). As this study primarily involved the collection of data from physicians, a web-based questionnaire was used as the data source in this study.

\section{Participants}

The plan was to include 480 physicians-both specialists and nonspecialists in a $1: 1$ ratiofrom eight different regions across Japan in the survey. Physicians who agreed to participate were sent a link to the survey and were screened further by asking them how many T2DM patients they had handled and the professional time they spent on patient care.

\section{Study Outcomes}

The primary endpoints were the proportions of physicians that considered various treatment factors (such as drug efficacy, tolerability, and other features) and patient characteristics when selecting the appropriate $\mathrm{OAD}$ for drug-naive patients with T2DM. The secondary endpoints included the proportions of physicians who selected particular OADs as the first-line therapy and the patient characteristics that influenced the treatment intensification decision in drugnaive patients with T2DM.

\section{Statistical Analysis}

A precision-based approach was used to evaluate the sample size, where approximately $40 \%$ of the physicians $(n=192)$ selected their most frequently prescribed drug. The proportion of physicians that considered a particular factor when selecting the OAD ranged from 20 to 50\%. The half-width of the $95 \%$ confidence interval (CI) was $4.7-5.8 \%$, which provided the range of $10 \%$ for the estimate. Categorical variables were presented as a number and proportion, whereas continuous variables were expressed as the mean, standard deviation, 25 th percentile, median, 75 th percentile, and the minimum and maximum values of the distribution as applicable.

To elucidate the reasons that the physicians chose the OADs, multinomial logistic regression was performed to assess the odds ratio (OR) and to compare the most and second most popular OADs used as first-line therapy. All analyses were performed using the Statistical Package for the Social Sciences (SPSS) (IBM ${ }^{\circledR}$ SPSS ${ }^{\circledR}$ Statistics, version 24).

\section{Ethics and Good Clinical Practice}

The study was conducted in accordance with the ethical guidelines for medical and health research involving human subjects as defined by the Ministry of Education, Culture, Sports, Science and Technology and the Ministry of Health, Labour and Welfare, Japan. We carried out the study in accordance with the code of professional behavior and relevant privacy principles. All physicians consented to be part of this survey and to have their data reported in this manuscript. The study protocol was reviewed and approved by a central ethics committee (EC) in Osaka.

\section{RESULTS}

\section{Background Characteristics of the Participating Physicians}

A total of 240 diabetes specialists and 251 nonspecialists participated in the online survey, 
which was conducted from 26 May to 26 June 2017. The geographical distributions of both groups across the eight selected regions in Japan were similar (Table 1). The background characteristics of the specialists and nonspecialists differed with respect to parameters such as age, practice setting, and medical specialty. The mean age of the specialists was $47.8 \pm 9.6$ years, with $16.5 \pm 8.7$ years of experience in clinical practice, whereas the mean age of the nonspecialists was $50.9 \pm 9.7$ years, and they had more experience in treating patients (21.9 \pm 9.4 years). The number of drug-naive T2DM patients treated by the physician in the last six months was similar for the specialists and nonspecialists, as was the percentage of their professional time spent on patient care (see Table 1).

\section{Choice of Initial Therapy for Drug-Naive Patients}

Among the various OADs available, dipeptidyl peptidase-4 inhibitors (DPP-4is) were used by the largest percentage of physicians, followed by metformin, regardless of whether specialists (DPP-4i: 69\%; metformin: 60\%) or nonspecialists (DPP-4i: 73\%; metformin: 47\%) were considered. Other drugs that were prescribed by the physicians included SGLT-2 inhibitors, which were more popular with nonspecialists (nonspecialists: $14 \%$ vs. specialists: $8 \%$ ), and glinides, which were more popular with specialists (specialists: 6\% vs. nonspecialists: 1\%) (Fig. 1). When the OAD most frequently used by each physician was considered, similar proportions of the specialists were found to most frequently prescribe DPP-4is (49\%) and metformin (45\%) to drug-naive patients as the first-line treatment, whereas a considerably larger percentage $(59 \%)$ of the nonspecialists most frequently prescribed DPP-4is as compared to those who most frequently prescribed metformin (34\%). SGLT-2 inhibitors (specialists: $3 \%$ vs. nonspecialists: $4 \%$ ), sulfonylureas (specialists: $2 \%$ vs. nonspecialists: $1 \%$ ), alpha-glucosidase inhibitors ( $1 \%$ for both specialists vs. nonspecialists), and glinides ( $1 \%$ for specialists) were only rarely the most frequently prescribed OADs (see Fig. 1).
Table 1 Background characteristics of the participating physicians

\begin{tabular}{lll}
\hline Parameter & $\begin{array}{l}\text { Specialists } \\
(\boldsymbol{n}=\mathbf{2 4 0})\end{array}$ & $\begin{array}{l}\text { Nonspecialist } \\
(\boldsymbol{n}=\mathbf{2 5 1})\end{array}$ \\
\hline Age (years) & $47.8 \pm 9.6$ & $50.9 \pm 9.7$ \\
\% of time spent on & $89.0 \pm 11.0$ & $90.2 \pm 9.9$ \\
patient care & & \\
Practice setting & & \\
Hospital-based & $188(78.3 \%)$ & $149(59.4 \%)$ \\
Office-based & $52(21.7 \%)$ & $102(40.6 \%)$ \\
Medical specialty & & \\
PCP/GP & $6(2.5 \%)$ & $48(19.1 \%)$ \\
Internist & $25(10.4 \%)$ & $121(48.2 \%)$ \\
Diabetologist & $189(78.8 \%)$ & $21(8.4 \%)$ \\
Endocrinologist & $16(6.7 \%)$ & $9(3.6 \%)$ \\
Cardiologist & $4(1.7 \%)$ & $52(20.7 \%)$ \\
Average number of & $16.5 \pm 8.7$ & $21.9 \pm 9.4$ \\
years spent practicing & & \\
Region & & \\
Hokkaido & $17(7.1 \%)$ & $15(6.0 \%)$ \\
Tohoku & $10(4.2 \%)$ & $16(6.4 \%)$ \\
Kanto & $76(31.7 \%)$ & $89(35.5 \%)$ \\
Chubu & $45(18.8 \%)$ & $38(15.1 \%)$ \\
Kinki & $33(13.8 \%)$ & $43(17.1 \%)$ \\
Chugoku & $13(5.4 \%)$ & $8(3.2 \%)$ \\
Shikoku & $17(7.1 \%)$ & $11(4.4 \%)$ \\
Kyushu & $29(12.1 \%)$ & $31(12.4 \%)$ \\
Number of drug-naive & $35.6 \pm 40.7$ & $35.1 \pm 63.1$
\end{tabular}

T2DM patients treated

in the last 6 months

The values presented are the mean \pm standard deviation or $n$ (\%) unless otherwise specified

$P C P$ primary care physician, $G P$ general practitioner, T2DM type 2 diabetes mellitus

Since both specialists and nonspecialists selected mainly DPP-4is or metformin as the first-line $\mathrm{OAD}$, we focused on these drugs in subsequent analyses. 


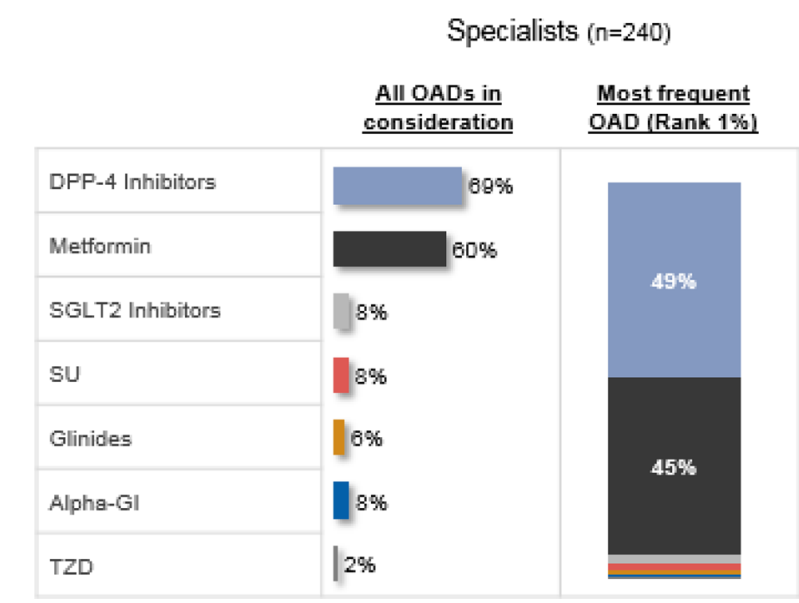

Fig. 1 Selection of the initial treatment for drug-naive patients with T2DM. Each value shown in the table is a proportion of the total physician population-either the proportion who have prescribed a particular OAD (the " $\%$ of physicians who prescribe the OAD” columns) or the proportion who prescribe this particular OAD more

\section{Treatment Factors Affecting the Selection of a DPP-4i or Metformin as the First-Line OAD}

\section{DPP-4 Inhibitors}

The treatment factors most commonly considered by specialists when prescribing DPP-4is were HbA1c-lowering effect, postprandial glucose (PPG)-lowering effect, a low risk of hypoglycemia, fasting plasma glucose (FPG)lowering effect, and no weight gain $(89 \%, 85 \%$, $83 \%, 69 \%$, and $68 \%$, respectively), whereas the corresponding proportions for nonspecialists were $94 \%, 86 \%, 87 \%, 74 \%$, and $70 \%$, respectively (Fig. 2a, b).

\section{Metformin}

Improvement in insulin resistance, low cost, low risk of hypoglycemia, and HbA1c- and FPGlowering effects were treatment factors that commonly influenced the selection of metformin by specialists $(93 \%, 91 \%, 89 \%, 87 \%$, and $85 \%$, respectively) and by nonspecialists (80\%, $92 \%, 81 \%, 86 \%$, and $75 \%$, respectively). Considerable scientific evidence (81\%) and PPGlowering effect $(75 \%)$ were other factors that significantly drove the selection of metformin by nonspecialists (Fig. 2a, b).
Non-specialists $(\mathrm{n}=251$ )

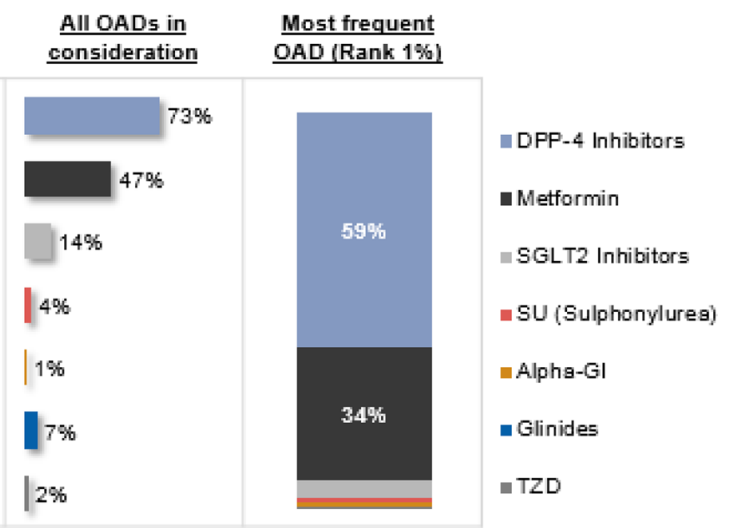

frequently than any other OADs (the "Most frequently prescribed OAD (\% of physicians)" columns). DPP-4 dipeptidyl peptidase-4, GI glucosidase inhibitor, $O A D s$ oral antidiabetes drugs, $S G L T-2$ sodium-glucose cotransporter2, $S U$ sulfonylurea, $T 2 D M$ type 2 diabetes mellitus, $T Z D$ thiazolidinedione

\section{Treatment Factors Affecting the Selection of the Initial OAD in Drug-Naive Patients: Comparing the Physicians' Choices}

There was a considerable difference of $>10 \%$ between diabetes specialists and nonspecialists in the importance of treatment factors such as a low risk of gastrointestinal side effects, improvement in insulin resistance, effect on glucagon, protection of $\beta$-cell function, and frequency of administration when DPP-4is were chosen (Fig. 2a, b).

Treatment factors with a more than $10 \%$ difference in influence between specialists and nonspecialists were effect on insulin, effect on glucagon, no weight gain, improvement in insulin resistance, and PPG-lowering effect when metformin was chosen as the first-line OAD (Fig. 2a, b).

\section{Regression Analysis Comparing DPP-4is and Metformin}

The ORs for the effects of various treatment factors when selecting DPP-4is or metformin as the first-line $\mathrm{OAD}$ are presented in Table 2. Among specialists, ease of use in patients with renal impairment (OR 11.7; 95\% CI 2.4, 57.3) 
(A)

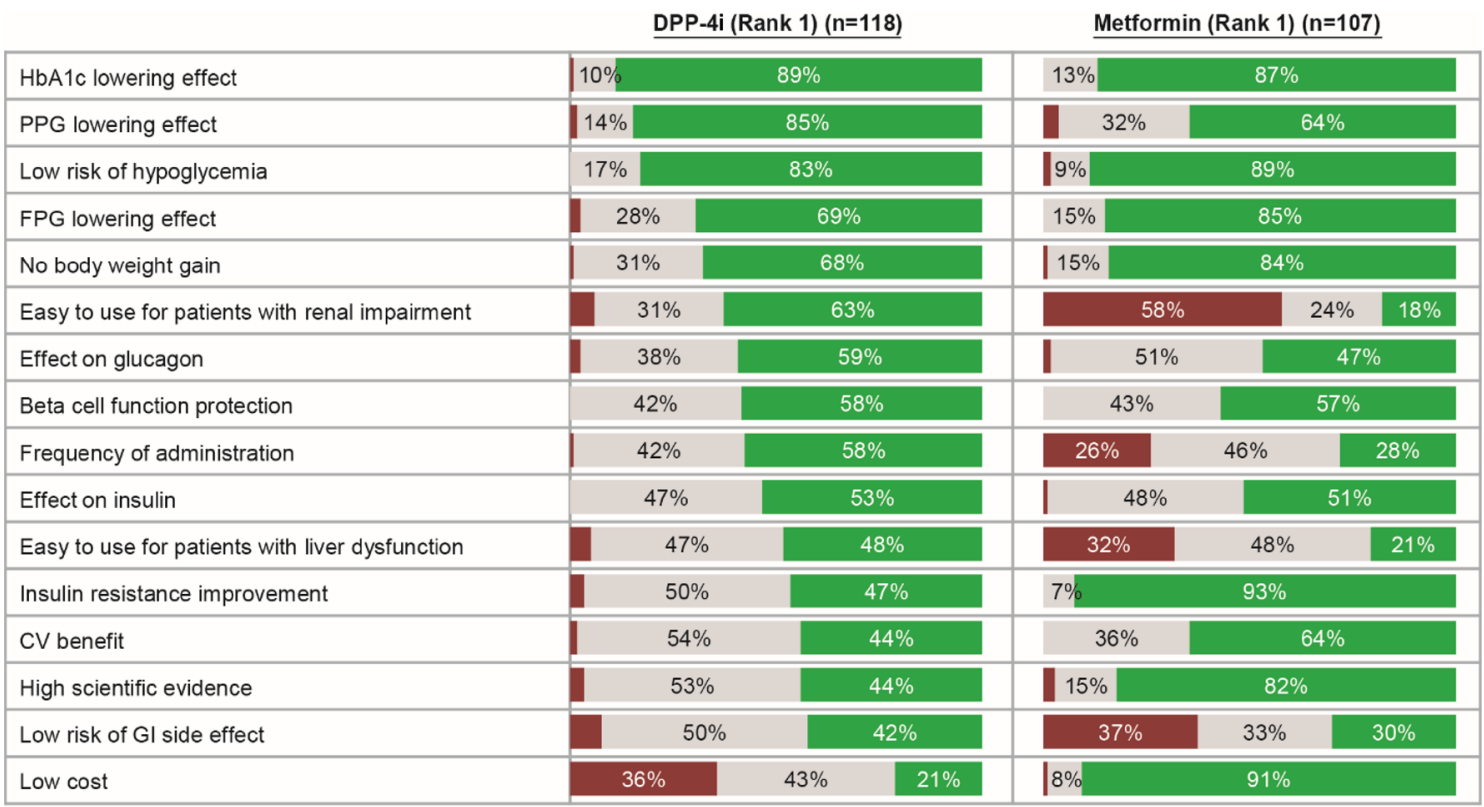

negative Impact $\quad$ Neutral $\quad$ Positive Impact

(B)

\begin{tabular}{|c|c|c|c|c|c|}
\hline & \multicolumn{2}{|c|}{ DPP-4i (Rank 1) $(n=148)$} & \multicolumn{3}{|c|}{ Metformin (Rank 1) ( $n=85)$} \\
\hline $\mathrm{HbA} 1 \mathrm{c}$ lowering effect & $5 \%$ & $94 \%$ & $13 \%$ & $86 \%$ & \\
\hline PPG lowering effect & $14 \%$ & $86 \%$ & $25 \%$ & $75^{\circ}$ & \\
\hline Low risk of hypoglycemia & $13 \%$ & $87 \%$ & $16 \%$ & $81 \%$ & \\
\hline FPG lowering effect & $24 \%$ & $74 \%$ & $25 \%$ & 75 & \\
\hline No body weight gain & $30 \%$ & $70 \%$ & $29 \%$ & & \\
\hline Easy to use for patients with renal impairment & $36 \%$ & $60 \%$ & $46 \%$ & & $14 \%$ \\
\hline Effect on glucagon & $51 \%$ & $49 \%$ & & & $25 \%$ \\
\hline Beta cell function protection & $30 \%$ & $68 \%$ & $45 \%$ & & \\
\hline Frequency of administration & $30 \%$ & $68 \%$ & $22 \%$ & $51 \%$ & $27 \%$ \\
\hline Effect on insulin & $53 \%$ & $46 \%$ & & & $27 \%$ \\
\hline Easy to use for patients with liver dysfunction & $51 \%$ & $49 \%$ & $24 \%$ & $61 \%$ & $15 \%$ \\
\hline Insulin resistance improvement & $40 \%$ & $58 \%$ & $19 \%$ & $80 \%$ & \\
\hline CV benefit & $52 \%$ & $45 \%$ & $40 \%$ & & \\
\hline High scientific evidence & $43 \%$ & $53 \%$ & $19 \%$ & $81 \%$ & \\
\hline Low risk of $\mathrm{Gl}$ side effect & $38 \%$ & $59 \%$ & $33 \%$ & $47 \%$ & $20 \%$ \\
\hline Low cost & $27 \%$ & $23 \%$ & $17 \%$ & $92 \%$ & \\
\hline
\end{tabular}

Fig. 2 Comparison of the importance of various treatment factors during the selection of a DPP-4i or metformin as the first-line OAD by specialists (a) and nonspecialists $(\mathbf{b})$. The values shown in the figure are percentages of physicians. $C V$ cardiovascular, DPP-4i dipeptidyl peptidase-4 inhibitor, $F P G$ fasting plasma glucose, GI gastrointestinal, $H b A 1 c$ glycated hemoglobin, $O A D$ oral antidiabetes drug, $P P G$ postprandial glucose 
Table 2 Effects of various treatment factors on the selection of a DPP-4i or metformin as the first-line drug of choice

\begin{tabular}{|c|c|c|c|c|}
\hline \multirow[t]{2}{*}{ Drug class } & \multirow[t]{2}{*}{ Treatment factor } & \multirow[t]{2}{*}{ OR } & \multicolumn{2}{|c|}{$95 \% \mathrm{CI}$} \\
\hline & & & Lower & Upper \\
\hline \multicolumn{5}{|l|}{ Specialists } \\
\hline \multirow[t]{2}{*}{$\begin{array}{l}\text { DPP-4 } \\
\text { inhibitors }\end{array}$} & $\begin{array}{l}\text { Easy to use for } \\
\text { patients with } \\
\text { renal } \\
\text { impairment }\end{array}$ & 11.7 & 2.4 & 57.3 \\
\hline & $\begin{array}{l}\text { Frequency of } \\
\text { administration }\end{array}$ & 8.6 & 1.9 & 38.6 \\
\hline Metformin & $\begin{array}{l}\text { Insulin resistance } \\
\text { improvement }\end{array}$ & 0.1 & 0.02 & 0.6 \\
\hline & Low cost & 0.02 & 0.003 & 0.08 \\
\hline \multicolumn{5}{|l|}{ Nonspecialists } \\
\hline \multirow[t]{2}{*}{$\begin{array}{l}\text { DPP-4 } \\
\text { inhibitors }\end{array}$} & $\begin{array}{l}\text { HbAlc-lowering } \\
\text { effect }\end{array}$ & 63.1 & 4.4 & 913.4 \\
\hline & $\begin{array}{l}\text { Easy to use for } \\
\text { patients with } \\
\text { renal } \\
\text { impairment }\end{array}$ & 10.7 & 1.7 & 67.8 \\
\hline \multirow[t]{2}{*}{ Metformin } & $\begin{array}{l}\text { Insulin resistance } \\
\text { improvement }\end{array}$ & 0.2 & 0.04 & 1.0 \\
\hline & Low cost & 0.01 & 0.001 & 0.05 \\
\hline
\end{tabular}

CI confidence interval, DPP-4 dipeptidyl peptidase- 4 , $H b A 1 c$ glycated hemoglobin, $O R$ odds ratio

and frequency of administration (OR 8.6; 95\% CI 1.9, 38.6) were the most influential factors when choosing DPP-4is. Alternatively, improvement in insulin resistance (OR 0.1; 95\% CI $0.02,0.6)$ and low cost (OR 0.02; 95\% CI $0.003,0.08$ ) were the factors driving the selection of metformin.

The nonspecialists mainly considered HbA1c-lowering effect (OR 63.1; 95\% CI 4.4, 913.4) and ease of use in patients with renal impairment (OR 10.7; 95\% CI 1.7, 67.8) as factors when selecting DPP-4is. The treatment factors that were most important to diabetes nonspecialists who selected metformin were the same as those of specialists: improvement in insulin resistance (OR $0.2 ; 95 \%$ CI $0.04,1.0)$ and low cost (OR 0.01; 95\% CI 0.001, 0.05).

\section{Patient Characteristics Affecting the Selection of DPP-4is or Metformin as First-Line OAD}

\section{DPP-4 Inhibitors}

The specialists and nonspecialists had similar considerations regarding patient characteristics. More than $50 \%$ of the physicians who prescribed a DPP-4i as the first-line OAD were most strongly influenced by PPG-lowering effect, followed by renal function (specialists: $56 \%$ and 53\%; nonspecialists: $51 \%$ and $57 \%$, respectively; see Fig. 3a, b).

\section{Metformin}

Both specialists and nonspecialists who selected metformin as the first-line OAD considered similar patient characteristics: age, renal function, BMI, and insulin resistance (specialists: 81\%, 76\%, 71\%, and 64\%; nonspecialists: 59\%, $71 \%, 75 \%$, and $56 \%$, respectively; see Fig. 3a, b). It should be noted, however, that age and insulin resistance were considered more by specialists than nonspecialists.

The influence of PPG-lowering effect on diabetes specialists was over $10 \%$ greater when DPP-4is were selected rather than metformin, whereas they were more strongly influenced by age, BMI, insulin resistance, and renal function (difference $>10 \%$ ) when metformin was selected over DPP-4is. A similar trend in the effects of the various patient characteristics was observed for nonspecialists who selected either DPP-4is or metformin as the first-line OAD.

\section{Patient Characteristics Affecting the Selection of the Initial OAD in Drug- Naive Patients: Comparing the Physicians' Choices}

When a DPP-4i was selected as the first-line $\mathrm{OAD}$, the average number of patient characteristics considered by a specialist was $4.3 \pm 2.8$, whereas the average number considered by a nonspecialist was $4.1 \pm 2.7$ ) The influence of BMI was more than $10 \%$ stronger among the 
(A)

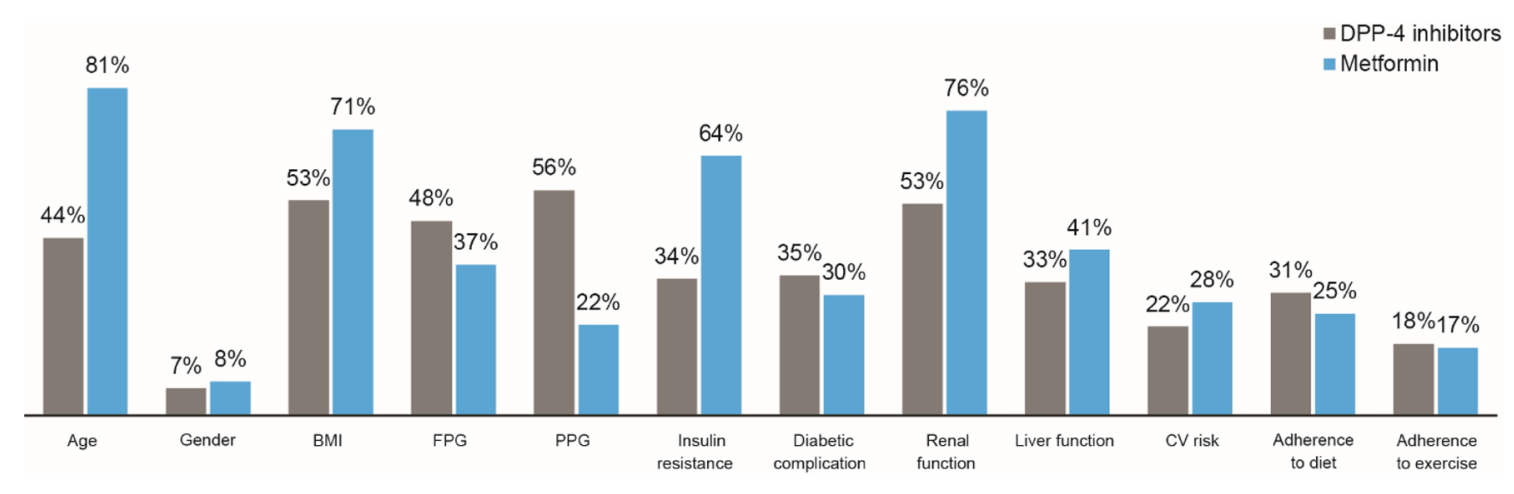

(B)

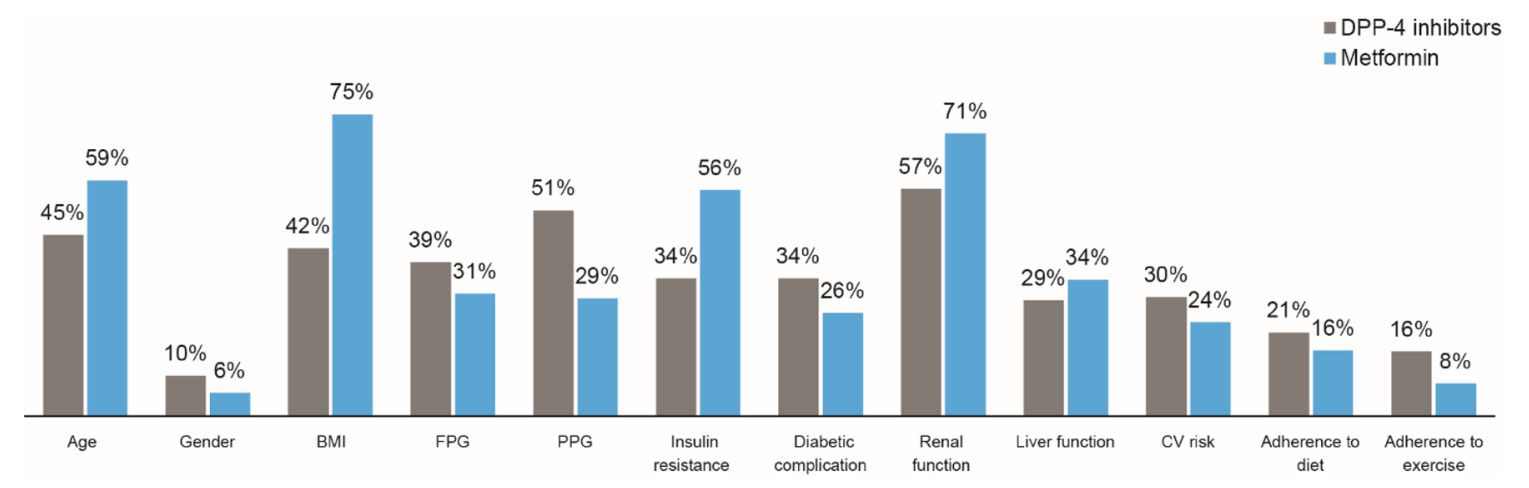

Fig. 3 Comparison of the effects of various patient characteristics on the selection of DPP-4i or metformin as the first-line OAD by specialists (a; DPP- $4 n=118$, metformin $n=107$ ) and nonspecialists (b; DPP- $4 n=$ 148 , metformin $n=85$ ). The values shown in the figure are the percentages of physicians whoconsidered the

specialists than the nonspecialists when a DPP$4 \mathrm{i}$ was chosen as the first-line OAD. When metformin was chosen as the first-line OAD, the average number of patient features considered was slightly higher for specialists than for nonspecialists $(5.0 \pm 2.8$ vs. $4.4 \pm 2.3)$, and the only patient characteristic that influenced diabetes specialists over 10\% more strongly than nonspecialists was age (Fig. 3a, b).

\section{Patient Characteristics Affecting the Treatment Intensification Decision}

The patient characteristics that were most influential in the treatment intensification patient characteristics when selecting a DPP-4i or metformin as the first-line OAD. BMI body mass index, $C V$ cardiovascular, DPP-4i dipeptidyl peptidase-4 inhibitor, $F P G$ fasting plasma glucose, $O A D$ oral antidiabetes drug, $P P G$ postprandial glucose

decision were similar to those considered when diabetes specialists and nonspecialists selected either DPP-4is or metformin as the first-line OAD in drug-naive T2DM patients (Figs. 3, 4).

\section{Frequency of Laboratory Tests for T2DM Patients}

Most specialists and nonspecialists responded that they measured body weight $(83.3 \%$ and $74.1 \%)$ and HbA1c (73.8\% and 62.2\%) every month, but the specialists were more regular with these tests than the diabetes nonspecialists were (Table 3). PPG, serum creatinine, and liver function parameters [aspartate transaminase 
(A)

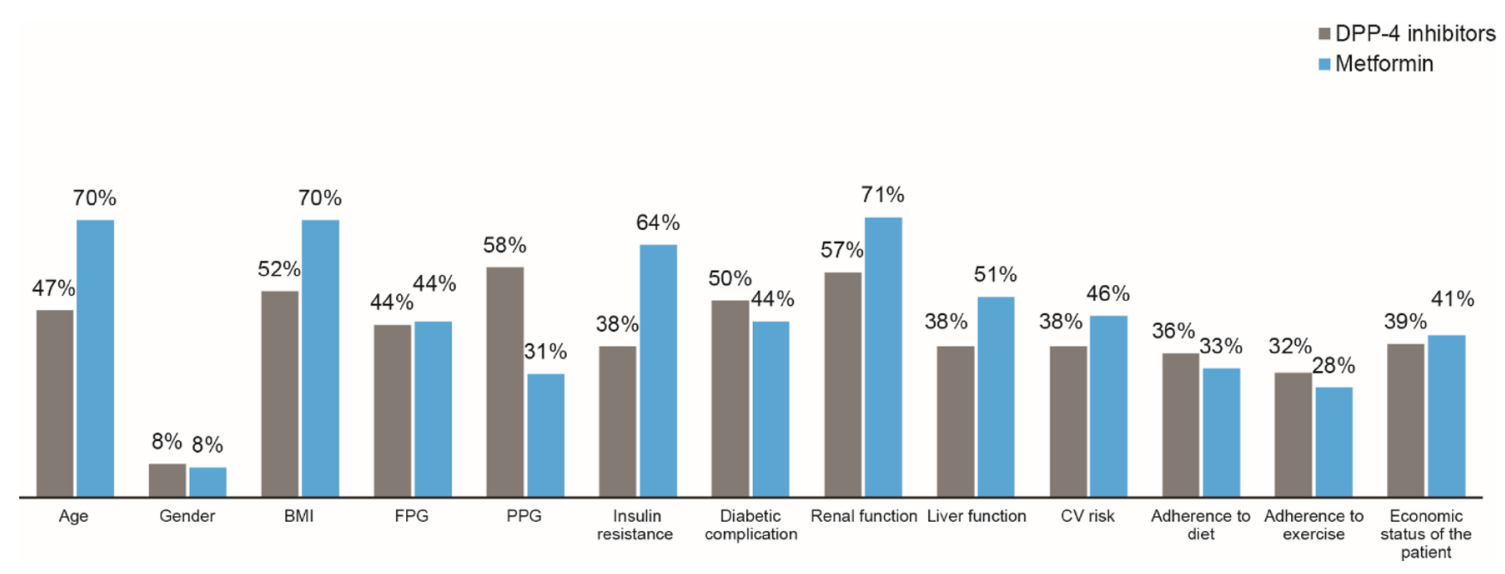

(B)

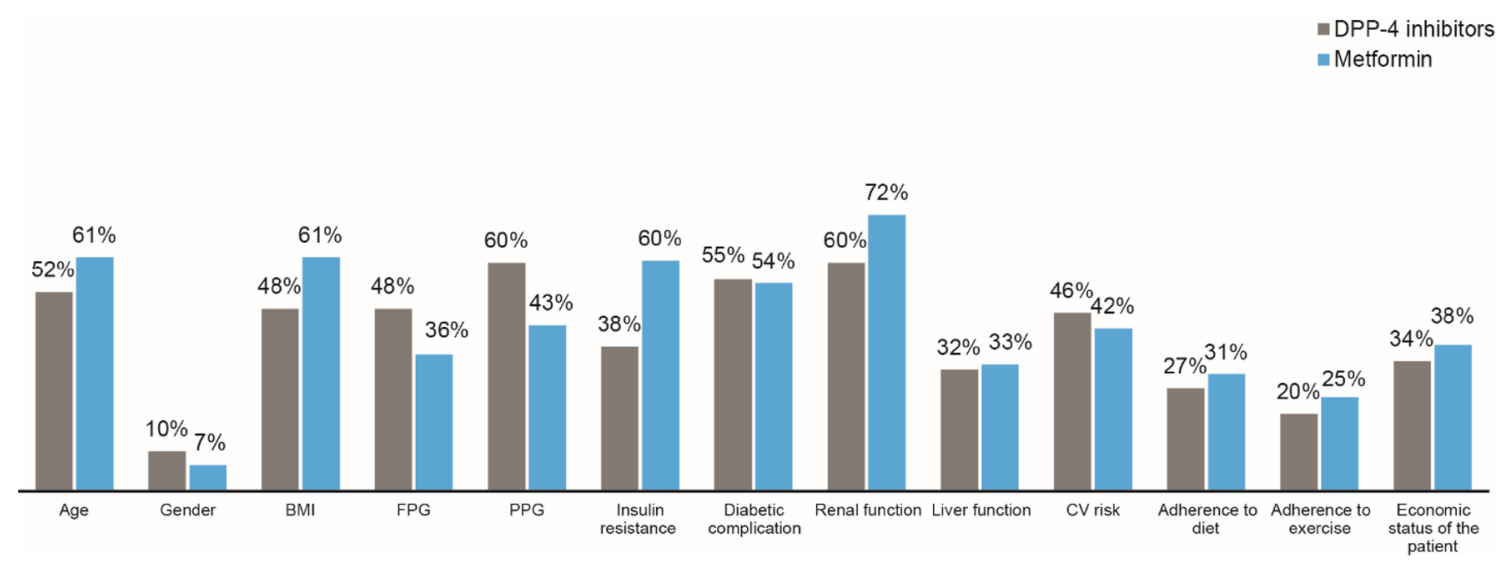

Fig. 4 a, b Comparison of the effects of various patient characteristics on the treatment intensification decision made by specialists (a; DPP-4 $n=165$, metformin $n=$ 144) and nonspecialists (b; DPP- $4 n=183$, metformin $n$ $=118$ ) for patients receiving DPP-4i or metformin as the first-line OAD. The values shown in the figure are the percentages of physicians whoconsidered the patient

(AST), alanine aminotransferase (ALT), and gamma-glutamyl transferase $(\gamma$-GTP)] were measured at least every 3 months by more than $75 \%$ of the physicians. However, the specialists measured these parameters more frequently than the nonspecialists did. The findings from the survey also indicated that around $45.0 \%$ of the specialists measured C-peptide every $7-12$ months, whereas $34.7 \%$ of the nonspecialists performed this test during the same period. Around $22-25 \%$ of the specialists and characteristics during the treatment intensification decision when a DPP-4i or metformin was employed as the first-line OAD. $B M I$ body mass index, $C V$ cardiovascular, $D P P-4 i$ dipeptidyl pepditase- 4 inhibitor, $F P G$ fasting plasma glucose, $O A D$ oral antidiabetes drug, $P P G$ postprandial glucose

nonspecialists responded that they measured the FPG at least every 2 months.

\section{Diabetes Complication Checks for Drug- Naive T2DM Patients}

It was found that $91.7 \%$ of the specialists conducted checks for diabetic retinopathy and $86.3 \%$ conducted checks for neuropathy in drug-naive patients; the corresponding percentages of the diabetes nonspecialists were 
Table 3 Data on the frequencies that various laboratory tests were ordered for T2DM patients by specialists and nonspecialists

\begin{tabular}{|c|c|c|c|c|c|c|c|}
\hline Frequency of the tests & $\begin{array}{l}\text { Body weight } \\
(\%)\end{array}$ & $\begin{array}{l}\text { HbAlc } \\
(\%)\end{array}$ & $\begin{array}{l}\text { FPG } \\
(\%)\end{array}$ & $\begin{array}{l}\text { PPG } \\
(\%)\end{array}$ & $\begin{array}{l}\text { Serum } \\
\text { creatinine (\%) }\end{array}$ & $\begin{array}{l}\operatorname{AST} / \operatorname{ALT} / \gamma- \\
\operatorname{GTP}(\%)\end{array}$ & $\begin{array}{l}\text { C-peptide } \\
(\%)\end{array}$ \\
\hline \multicolumn{8}{|l|}{ Specialists } \\
\hline $\begin{array}{l}\text { Do not perform this } \\
\text { test }(0.0)\end{array}$ & 0.4 & 0.4 & 13.3 & 4.2 & 0.4 & 1.3 & 22.1 \\
\hline $\begin{array}{l}\text { Every } 7-12 \text { months } \\
\quad(1.5)\end{array}$ & 0.0 & 0.8 & 7.1 & 1.3 & 1.7 & 2.5 & 45.0 \\
\hline $\begin{array}{l}\text { Every } 4-6 \text { months } \\
\quad(2.5)\end{array}$ & 0.8 & 0.4 & 10.4 & 5.4 & 11.7 & 13.8 & 15.0 \\
\hline Every 3 months (4.0) & 5.0 & 5.4 & 12.5 & 12.9 & 19.2 & 18.3 & 11.3 \\
\hline Every 2 months (6.0) & 10.4 & 19.2 & 25.0 & 27.5 & 21.7 & 22.5 & 3.8 \\
\hline Every month (12.0) & 83.3 & 73.8 & 31.7 & 48.8 & 45.4 & 41.7 & 2.9 \\
\hline \multicolumn{8}{|l|}{ Nonspecialists } \\
\hline $\begin{array}{l}\text { Do not perform this } \\
\text { test }(0.0)\end{array}$ & 2.0 & 0.0 & 13.9 & 11.2 & 0.4 & 2.4 & 35.1 \\
\hline $\begin{array}{l}\text { Every } 7-12 \text { months } \\
\quad(1.5)\end{array}$ & 1.2 & 0.0 & 3.6 & 1.2 & 2.4 & 3.6 & 34.7 \\
\hline $\begin{array}{l}\text { Every } 4-6 \text { months } \\
\quad(2.5)\end{array}$ & 2.8 & 2.4 & 8.8 & 7.2 & 21.1 & 20.7 & 16.7 \\
\hline Every 3 months (4.0) & 7.6 & 12.7 & 16.7 & 13.9 & 24.7 & 26.3 & 8.0 \\
\hline Every 2 months (6.0) & 12.4 & 22.7 & 22.3 & 24.3 & 23.9 & 23.9 & 5.2 \\
\hline Every month (12.0) & 74.1 & 62.2 & 34.7 & 42.2 & 27.5 & 23.1 & 0.4 \\
\hline
\end{tabular}

The values presented are the percentages of the diabetes specialists and nonspecialists who prescribed the tests $\gamma$-GTP gamma-glutamyl transferase, $A L T$ alanine aminotransferase, $A S T$ aspartate transaminase, $F P G$ fasting plasma glucose, $H b A l c$ glycated hemoglobin, $P P G$ postprandial glucose

$80.1 \%$ and $61.0 \%$, respectively. However, almost all specialists and nonspecialists examined the patients for renal complications (97.5\% vs. 96.4\%; see Fig. 5).

\section{DISCUSSION}

The present study explored the influences of various treatment factors and patient characteristics on physicians (diabetes specialists and nonspecialists) when they select the first-line treatment for drug-naive patients with T2DM in a real-world setting in Japan.
It is interesting to note that the numbers of drug-naive patients with T2DM treated over the last six months by specialists and nonspecialists were similar. This can be explained by the fact that the number of specialists for the large Japanese T2DM population of about 7.4 million in 2017 was approximately 5500 [3]. As the number of people with T2DM is increasing, there is also an increased demand for specialists, which in turn may be leading to an increasing number of visits to nonspecialists.

Our questionnaire-based survey of physicians revealed that the first-line OADs most 


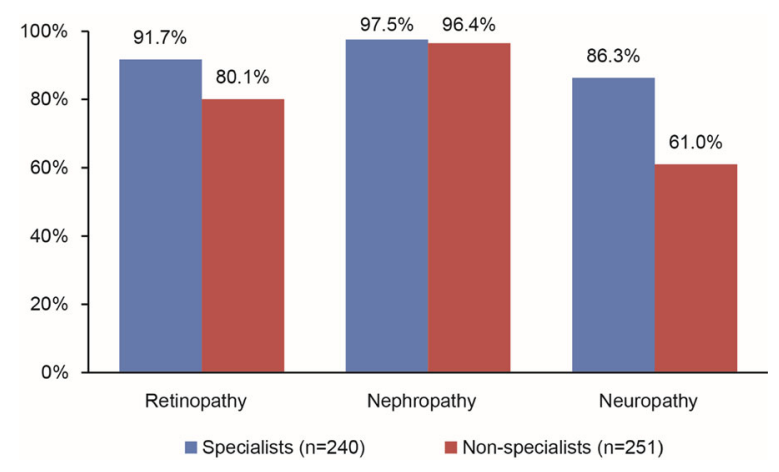

Fig. 5 Percentages of the specialists and nonspecialists who performed checks for various diabetes complications. The values shown in the figure are the percentages of physicians whochecked for the particular diabetes complications in drug-naivepatients with T2DM

frequently prescribed by specialists and nonspecialists in Japan were DPP-4is, followed by metformin. HbA1c-lowering effect and a low risk of hypoglycemia were considered to be among the most important treatment factors when a DPP-4i or metformin was selected as the first-line OAD. However, the influences of treatment factors such as ease of use in patients with renal impairment, improvement in insulin resistance, and low cost on the first-line OAD selection process depended significantly on whether a DPP-4i or metformin was chosen. According to multinomial logistic regression analysis, the selection of a DPP-4i over metformin was dependent on the importance to specialists of the ease of use of the drug in patients with renal impairment and the frequency of administration, or the importance of the HbA1c-lowering effect to nonspecialists. When metformin was preferred, this was due to attributes such as improvement in insulin resistance and cost effectiveness. While factors influencing the selection of DPP-4is or metformin were similar for specialists and nonspecialists, the former considered the frequency of administration while the latter considered HbA1c-lowering effect as a factor when selecting a DPP-4i rather than metformin. Though the reason for this is not clear, it may be related to the dosage of metformin prescribed by the nonspecialists; the lower dose of metformin $(500 \mathrm{mg}$ ) prescribed by the nonspecialists may have resulted in a suboptimal HbA1c-lowering effect of metformin compared to that of the DPP-4is. The assignment of higher dosages by specialists may have resulted in similar HbA1clowering effects of DPP-4is and metformin, meaning that the frequency of administration was left as a factor by which to select DPP-4is according to regression analysis.

The underlying reason for the choice of a DPP- $4 \mathrm{i}$ as the first-line OAD in drug-naive T2DM patients could be the variability in the pathophysiology of T2DM in East Asians, including the Japanese population. It is well established that T2DM in East Asians is characterized by $\beta$-cell dysfunction; incretin-based therapies such as DPP-4is most likely exert their glucose-lowering effects by improving $\beta$-cell dysfunction, as they increase the concentrations of active GLP-1 and glucose-dependent insulinotropic polypeptides (GIP) [16]. The stronger HbA1c-lowering effects of incretinbased therapies such as DPP-4i in East Asians compared with Caucasians may further confirm that $\beta$-cell dysfunction is a greater influence on hyperglycemia in the former group [17]. Additionally, the availability of DPP-4is as first-line monotherapies in Japan based on Japanese guidelines [3] makes them more attractive choices for use as the initial drug therapy in newly diagnosed patients with T2DM. It is also important to note that metformin was considered by both specialists and nonspecialists due to its ability to improve insulin resistance in patients with T2DM and its cost effectiveness. The finding that metformin is one of the two most prevalent initial prescriptions suggests that the recommendations by the American Diabetes Association (ADA) and The European Association for the Study of Diabetes (EASD) [9] that metformin should be used as the first-line OAD are generally followed in Japan.

The guidelines in Japan recommend that treatment objectives should be established on a case-by-case basis, considering parameters such as age, duration of disease, complications, risk of hypoglycemia, and support systems $[3,18]$. It is interesting to note that PPG-lowering effect was considered when the physician selected a DPP-4i, whereas age, BMI, improvement in insulin resistance, and renal function were considered when metformin was selected as the 
initial drug therapy by specialists as well as nonspecialists. This accentuates the fact that there is agreement between the diabetes specialists and nonspecialists in the patient characteristics that should be considered when choosing the first-line OAD for newly diagnosed T2DM patients. Age was less likely to be considered by nonspecialists who chose metformin as the first-line therapy than if they chose DPP4 is, but none of the other patient characteristics significantly differed in influence depending on whether a DPP-4i or metformin was selected. This observation can be explained by differences in the metformin dosages assigned by specialists and nonspecialists, as discussed above; if nonspecialists tend to prescribe a lower dose of metformin, they are unlikely to consider age as a factor due to the associated GI or lactic acidosis issues when prescribing metformin. However, there is no clear evidence to support this theory, so further investigations are warranted. In addition, non-specialists tend to consider BMI more than specialists while selecting metformin over DPP-4is. This could be explained by the findings of UKPDS 34, a wellknown study even of non-specialists, which indicated that metformin can decrease the risk of diabetes-related complications in overweight patients and is associated with weight neutrality and fewer hypoglycemic events [19].

The patient characteristics that tended to influence the treatment intensification decision were similar to those that most strongly influenced the selection of the first-line OAD by specialists. PPG-lowering effect was considered an important factor when a DPP-4i was prescribed, whereas age, renal function, BMI, and improvement in insulin resistance were the factors considered when metformin was chosen.

Substantial numbers of specialists and nonspecialists responded that they measured HbA1c monthly and C-peptide at least yearly. These test frequencies could be specific to clinical practice in Japan and may differ in other countries [20]. Such frequencies could depend on whether the health insurance system covers these laboratory tests in the country of interest.

At the time of the first consultation, the Japanese guidelines advise physicians to test for diabetes-related complications that may be present in these patients due to a delayed diagnosis, as it is essential to treat such complications [3]. In the present survey, although most of the parameters relating to diabetes and its complications were monitored by the physicians, we observed that kidney-related complications were reviewed to similar extents by both specialists and nonspecialists, indicating that nephropathy is an important concern among all physicians who treat T2DM patients in Japan.

The limitations of the present study should be considered. This study is noninterventional in nature and may include information bias, selection bias, and feasibility limitations. For example, we did not include the timing of administration but we did include the frequency of administration and the drug dosage. To be noted, participating physicians would respond their prescription preference based on general perception on DPP-4is rather than focusing on drug-naive patients whose renal function are usually not impaired. We must therefore be careful when applying these results in clinical practice because we believe that renal function is a critical factor in the decision of whether to use a DPP-4i or metformin. Diabetes complication checks were conducted frequently by both specialists and nonspecialists. This observation differs from previous reports [21], probably because no clear definition on diabetes complications, such as microalbuminuria was set, and therefore physicians could respond as they usually check diabetic nephropathy when they measure serum creatinine routinely. The behavior of physicians according to the current survey results should be checked for validity by comparing the results of this survey with other data sources such as databases in order to examine the consistency and differences between the data sources. Despite these limitations, web-based online surveys are considered to be a fast and cost-effective method of obtaining feedback from physicians (specialists and nonspecialists) spread across various geographical regions. They also facilitate the inclusion of both hospital-based and officebased specialists and nonspecialists. Additionally, the present survey length is capped at $25 \mathrm{~min}$ in order to maximize respondent 
participation and minimize the dropout rate. Also, we only focused on DPP-4is and metformin in this survey in order to examine the main factors affecting initial OAD selection for drug-naive patients, as these were the drugs predominantly prescribed by the physicians. Further studies are needed to examine other drugs, such as SGLT-2 inhibitors and sulfonylurea, to further probe the factors that affect the selection of drugs for more sophisticated diabetes treatments.

\section{CONCLUSION}

In summary, the findings from the present survey show that DPP-4is are the preferred firstline treatment by physicians in Japan due to their ease of use in patients with renal impairment, frequency of administration, and HbA1clowering effect. The next most popular first-line treatment is metformin, due to the resulting improvement in insulin resistance and its cost effectiveness. The patient characteristics taken into account when choosing the first-line OAD as well as when deciding upon treatment intensification were essentially the same regardless of whether the physician was a diabetes specialist or nonspecialist. In this regard, the information provided by this study should prompt discussion of the differences in T2DM treatment between Japan and other counties.

\section{ACKNOWLEDGEMENTS}

We thank the participants of the study.

Funding. Sponsorship and article processing charges for this study were funded by Novartis. All authors had full access to all of the data in this study and take complete responsibility for the integrity of the data and accuracy of the data analysis.

Medical Writing and/or Editorial Assistance. The authors thank Megha Saraf of Novartis Healthcare Pvt. Ltd, Hyderabad, India for her support when conducting the survey reported in this manuscript. The authors also thank Ishita
Guha Thakurta, Ph.D., of Novartis Healthcare Pvt. Ltd, Hyderabad, India for medical writing support, which was funded by Novartis Pharma AG, Basel, Switzerland, in accordance with good publication practice (GPP3) guidelines (http://www. ismpp.org/gpp3).

Authorship. All named authors meet the International Committee of Medical Journal Editors (ICMJE) criteria for authorship for this manuscript, take responsibility for the integrity of the work as a whole, and have given final approval to the version to be published.

Disclosures. Hiroki Murayama is an employee of Novartis Pharma K.K. Kota Imai is an employee of Novartis Pharma K.K. Masato Odawara has served as an advisory board member for Novartis, has received research grants with contracts from Novo Nordisk and Astellas, has received unrestricted research grants from Daiichi Sankyo, MSD, Ono, Novartis, Astellas, Sanwa Kagaku Kenkyusho, AstraZeneca, Kyowa Hakko Kirin, Kowa, Takeda, Mitsubishi Tanabe, Eli Lilly, Nippon Boehringer, Sanofi, Novo Nordisk, Sumitomo Dainippon, and Taisho Toyama, and has received lecture fees from Daiichi Sankyo, MSD, Ono, Novartis, Astellas, Sanwa Kagaku Kenkyusho, AstraZeneca, Kyowa Hakko Kirin, Kowa, Takeda, Mitsubishi Tanabe, Eli Lilly, Nippon Boehringer, Sanofi, Novo Nordisk, Sumitomo Dainippon, and Taisho Toyama.

Compliance with Ethics Guidelines. The study was conducted in accordance with the ethical guidelines for medical and health research involving human subjects defined by the Ministry of Education, Culture, Sports, Science and Technology and the Ministry of Health, Labour and Welfare, Japan. The study protocol was reviewed and approved by a central ethics committee (EC). All physicians who provided information for this survey consented to be part of it and to the inclusion of their survey data in a manuscript to be published in a peer-reviewed journal.

Data Availability. The data sets generated during and/or analyzed during the current 
study are available from the corresponding author on reasonable request.

Open Access. This article is distributed under the terms of the Creative Commons Attribution-NonCommercial 4.0 International License (http://creativecommons.org/licenses/ by-nc/4.0/), which permits any noncommercial use, distribution, and reproduction in any medium, provided you give appropriate credit to the original author(s) and the source, provide a link to the Creative Commons license, and indicate if changes were made.

\section{REFERENCES}

1. International Diabetes Federation. IDF diabetes atlas. 8th ed. Brussels: International Diabetes Federation; 2015. http://www.diabetesatlas.org. Accessed 29 Jan 2018.

2. Wild S, Roglic G, Green A, Sicree R, King H. Global prevalence of diabetes: estimates for the year 2000 and projections for 2030. Diabetes Care. 2004;27:1047-53.

3. Japan Diabetes Society, editor. Treatment guide for diabetes 2014-2015. Tokyo: Bunkodo; 2013. http:// www.jds.or.jp/modules/en/index.php?content_id= 1. Accessed 29 Jan 2018.

4. Kobayashi M, Yamazaki K, Hirao K, et al. The status of diabetes control and antidiabetic drug therapy in Japan-a cross-sectional survey of 17,000 patients with diabetes mellitus (JDDM 1). Diabetes Res Clin Pract. 2006;73:198-204.

5. Takahashi E, Moriyama K, Yamakado M, et al. Lifestyle and glycemic control in Japanese adults receiving diabetes treatment: an analysis of the 2009 Japan Society of Ningen Dock database. Diabetes Res Clin Pract. 2014;104:e50-3.

6. $\mathrm{Hu} \mathrm{H}$, Hori A, Nishiura $\mathrm{C}$, et al. HbA1c, blood pressure, and lipid control in people with diabetes: Japan Epidemiology Collaboration on Occupational Health study. PLoS ONE. 2016;11:e0159071.

7. Lavernia F, Adkins SE, Shubrook JH. Use of oral combination therapy for type 2 diabetes in primary care: meeting individualized patient goals. Postgrad Med. 2015;127:808-17.

8. Kohro $\mathrm{T}$, Yamazaki $\mathrm{T}$, Sato $\mathrm{H}$, et al. Trends in antidiabetic prescription patterns in Japan from 2005 to 2011. Int Heart J. 2013;54:93-7.
9. Inzucchi SE, Bergenstal RM, Buse JB, et al. Management of hyperglycemia in type 2 diabetes, 2015: a patient-centered approach: update to a position statement of the American Diabetes Association and The European Association for the Study of Diabetes. Diabetes Care. 2015;38:140-9.

10. Rafaniello C, Arcoraci V, Ferrajolo C, et al. Trends in the prescription of antidiabetic medications from 2009 to 2012 in a general practice of Southern Italy: a population-based study. Diabetes Res Clin Pract. 2015;108:157-63.

11. Montvida O, Shaw J, Atherton JJ, Stringer F, Paul SK. Long-term trends in antidiabetes drug usage in the U.S.: real-world evidence in patients newly diagnosed with type 2 diabetes. Diabetes Care. 2018;41:69-78.

12. Grant RW, Wexler DJ, Watson AJ, et al. How doctors choose medications to treat type 2 diabetes: a national survey of specialists and academic generalists. Diabetes Care. 2007;30:1448-53.

13. Filion KB, Joseph L, Boivin JF, Suissa S, Brophy JM. Trends in the prescription of anti-diabetic medications in the United Kingdom: a population-based analysis. Pharmacoepidemiol Drug Saf. 2009;18:973-6.

14. Hampp C, Borders-Hemphill V, Moeny DG, Wysowski DK. Use of antidiabetic drugs in the U.S., 2003-2012. Diabetes Care. 2014;37:1367-74.

15. Oishi M, Yamazaki K, Okuguchi F, et al. Changes in oral antidiabetic prescriptions and improved glycemic control during the years 2002-2011 in Japan (JDDM32). J Diabetes Investig. 2014;5:581-7.

16. Yabe D, Seino Y, Fukushima M, Seino S. $\beta$ cell dysfunction versus insulin resistance in the pathogenesis of type 2 diabetes in East Asians. Curr Diabetes Rep. 2015;15:36.

17. Kim YG, Hahn S, Oh TJ, Kwak SH, Park KS, Cho YM. Differences in the glucose-lowering efficacy of dipeptidyl peptidase- 4 inhibitors between Asians and non-Asians: a systematic review and metaanalysis. Diabetologia. 2013;56:696-708.

18. Araki E, Haneda M, Kasuga M, et al. New glycemic targets for patients with diabetes from the Japan Diabetes Society. J Diabetes Investig. 2017;8:123-5.

19. UK Prospective Diabetes Study Group. Effect of intensive blood-glucose control with metformin on complications in overweight patients with type 2 diabetes (UKPDS 34). The Lancet. 1998;352:854-65.

20. Canadian Agency for Drugs and Technologies in Health. Rapid response report for Sept 2014. 
Ottawa: Canadian Agency for Drugs and Technologies in Health; 2014.

21. Yokoyama H, Kawai K. Kobayashi M; Japan Diabetes Clinical Data Management Study Group.
Microalbuminuria is common in Japanese type 2 diabetic patients: a nationwide survey from the Japan Diabetes Clinical Data Management Study Group (JDDM 10). Diabetes Care. 2007;30:989-92. 